\section{Optimum Weighted Smoothing in Finite Data}

K. C. Indukumar and V. U. Reddy

\begin{abstract}
In this correspondence, we consider a generalized smoothing problem and develop a procedure to obtain a set of optimum weights which gives minimum mean-squared error (MSE) in the estimates of directions of arrival of signals in finite data when the signals are arbitrarily correlated. Using the optimum weights, we study the optimum tradeoff between the number of subarrays and the subarray size for a fixed total size of the array. The computation of optimum weights, however, requires full knowledge of the scenario. Since exact DOA's, powers, and correlations of signals are unknown a priori, we give a method to estimate these weights from the observed finite data. We also show through empirical studies that the optimum weights can be approximated with Taylor weights which serve as near-optimum weights. Simulation results are included to support the theoretical assertions.
\end{abstract}

\section{INTRODUCTION}

The performance of eigenstructure based direction-of-arrival (DOA) estimation algorithms degrades in finite data in the presence of both correlated and uncorrelated sources. In the case of infinite data with fully correlated sources, the degradation is due to the collapse of the signal subspace, while in the case of finite data only, it is due to subspace perturbation. In the presence of correlated sources in finite data, on the other hand, the effective correlation between the signals affects the sensitivity between the subspaces; as the correlation between the signals increases, the performance degradation increases. Spatial smoothing is usually suggested to recover the collapsed signal subspace [1], and to reduce perturbations and sensitivity in the subspaces [2]. The performance of several DOA estimation algorithms, with and without spatial smoothing, has been studied in [3], [8], [6], [4]. In [4], [8], the authors have analyzed the finite data performance of MUSIC and minimum norm methods with spatial smoothing and derived expressions for the mean-squared error (MSE) in the DOA estimates.

In this correspondence, we consider a generalized smoothing (weighting) problem and obtain optimum weights which give minimum MSE in the DOA estimates. The optimization problem is formulated as a constrained minimization of the MSE function. The computation of optimum weights requires full knowledge of the scenario. Here, we suggest methods to estimate these weights and show through empirical results that precise knowledge of the scenario is not crucial. Though we consider root-MUSIC in our study, the analysis extends in a straightforward manner to other methods such as root-minimum norm.

\section{BACKGROUND}

Consider a linear equispaced array formed by $K$ overlapping subarrays with $M$ identical and omnidirectional sensors each. Assume that signals from $D$ far-field narrow-band emitters, denoted by $s_{1}(t), s_{2}(t), \cdots, s_{D}(t)$, are impinging on the array from directionsof-arrival (DOA's), $\theta_{1}, \theta_{2}, \cdots, \theta_{D}$, measured with respect to the

Manuscript received May 19, 1992; revised November 23, 1992. The associate editor coordinating the review of this correspondence and approving it for publication was Dr. K. M. Buckley.

The authors are with the Department of Electrical Communication Engineering, Indian Institute of Science, Bangalore 560 012, India.

IEEE Log Number 9208214. normal to the array. The signals are assumed to be zero-mean, stationary, and complex Gaussian random processes and the additive noise is assumed to be be a zero-mean, stationary and white complex Gaussian random process that is uncorrelated from sensor to sensor and is independent of the signals.

The data covariance matrix obtained from the data collected at the output of $l$ th subarray is given by [1]

$$
\boldsymbol{R}^{\prime}=\boldsymbol{A} \boldsymbol{\Phi}^{(l-1)} \boldsymbol{S} \boldsymbol{\Phi}^{-(l-1)} \boldsymbol{A}^{H}+\sigma_{n}^{2} \boldsymbol{I}
$$

where $\boldsymbol{A}$ denotes the direction matrix with its columns given by $\boldsymbol{a}\left(\theta_{i}\right)=\left[1, \exp \left(j(2 \pi d / \lambda) \sin \theta_{i}\right), \cdots, \exp (j(2 \pi d / \lambda)(M-1)\right.$ $\left.\left.\sin \theta_{i}\right)\right]^{T}, i=1,2, \cdots, D . \Phi$ is a diagonal matrix with $\exp$ $\left(j(2 \pi d / \lambda) \sin \theta_{1}\right), \cdots, \exp \left(j(2 \pi d / \lambda) \sin \theta_{D}\right)$ as its diagonal elements. $S$ is the source covariance matrix, $\sigma_{n}^{2}$ is the sensor noise power, $d$ is the interelement spacing, and $\lambda$ is the wavelength of the incidence wave.

In spatial smoothing (uniform weighting), the smoothed covariance matrix is computed as $\boldsymbol{R}=(1 / K) \Sigma_{i=1}^{K} \boldsymbol{R}^{\prime}$. The eigendecomposition of the matrix $\boldsymbol{R}$ is given by

$$
\boldsymbol{R}=\left[\boldsymbol{E}_{S} \mid \boldsymbol{E}_{N}\right]\left(\begin{array}{cc}
\left(\boldsymbol{\Lambda}_{S}+\sigma_{n}^{2} \boldsymbol{I}\right) & 0 \\
0 & \sigma_{n}^{2} \boldsymbol{I}
\end{array}\right)\left[\begin{array}{l}
\boldsymbol{E}_{S}^{H} \\
\boldsymbol{E}_{N}^{H}
\end{array}\right]
$$

where the span of $\boldsymbol{E}_{S}$, formed by the set of orthonormal eigenvectors corresponding to the first $D$ ordered eigenvalues, is referred to as the signal subspace and the span of $\boldsymbol{E}_{N}$, formed by the orthonormal eigenvectors corresponding to the last $(M-D)$ eigenvalues, as the noise subspace. $\boldsymbol{\Lambda}_{S}$ is a diagonal matrix with $D$ signal eigenvalues as the diagonal elements. Since the signal subspace is also spanned by the direction vectors it follows that

$$
\boldsymbol{a}\left(\theta_{i}\right)^{H} \boldsymbol{E}_{N} \boldsymbol{E}_{N}^{H} \boldsymbol{a}\left(\theta_{i}\right)=0 \quad \forall i=1, \cdots, D
$$

where $(\cdot)^{H}$ denotes Hermitian operation.

\section{Root-MUSIC [9]}

For a uniform linear array, $\boldsymbol{a}\left(\theta_{i}\right)$ can be denoted by $[1, z, \cdots$, $\left.z^{M}\right]^{T}$, where $z=\exp \left[j(2 \pi d / \lambda) \sin \theta_{i}\right]$. Now, solving (3) for $\theta_{i}$, $i=1, \cdots, D$, is equivalent to determining $D$ roots of the polynomial

$$
F(z)=\sum_{l=-(M-1)}^{M-1} b_{l} z^{-l}
$$

where the coefficient $b_{l}$ is the sum of entries along the $l$ th diagonal of the matrix $\boldsymbol{E}_{N} \boldsymbol{E}_{N}^{H}$. In the finite data case, we choose $D$ roots that lie closest to the unit circle and compute the DOA's from the roots.

Recently, an expression for the MSE in the estimated DOA's in finite data with spatial smoothing is developed in [4]. The expression is given by

$$
\overline{\left|\Delta \theta_{i}\right|^{2}}=\left(\frac{\lambda}{2 \pi d \cos \theta_{i}}\right)^{2} \frac{\left(\boldsymbol{\Gamma}_{\alpha_{i} \alpha_{i} \beta_{i} \beta_{i}}+\operatorname{Re} \boldsymbol{\Gamma}_{\alpha_{i} \beta_{i} \alpha_{i} \beta_{i}}\right)}{2\left(\boldsymbol{v}_{1}^{H}\left(\omega_{i}\right) \boldsymbol{P}_{N} \boldsymbol{v}_{1}\left(\omega_{i}\right)\right)^{2}}
$$

where the terms are defined as follows:

$$
\begin{aligned}
\boldsymbol{P}_{N} & =\boldsymbol{E}_{N} \boldsymbol{E}_{N}^{H} \\
\boldsymbol{v}(\omega) & =\frac{1}{\sqrt{M}}[1, \exp (j \omega), \cdots, \exp (j(M-1) \omega)]^{T} \\
\boldsymbol{v}_{1}\left(\omega_{i}\right) & =\left.\frac{\partial \boldsymbol{v}(\omega)}{\partial \omega}\right|_{\omega=\omega_{i}}, \quad \boldsymbol{a}_{i}^{H}=\boldsymbol{v}_{1}^{H}\left(\omega_{i}\right) \boldsymbol{P}_{N}
\end{aligned}
$$




$$
\begin{aligned}
\boldsymbol{\beta}_{i} & =\left(\frac{1}{K} \sum_{l=1}^{K} \boldsymbol{A} \boldsymbol{\Phi}^{(l-1)} \boldsymbol{S} \boldsymbol{\Phi}^{-(l-1)} \boldsymbol{A}^{H}\right)^{\#} \boldsymbol{v}\left(\omega_{i}\right) \\
\boldsymbol{\Gamma}_{\alpha_{i} \alpha_{i} \beta_{i} \beta_{i}} & =\frac{1}{N K^{2}} \sum_{p=1}^{K} \sum_{q=1}^{K}\left(\boldsymbol{\alpha}_{i}^{H} \boldsymbol{R}_{p q} \boldsymbol{\alpha}_{i}\right)\left(\boldsymbol{\beta}_{i}^{H} \boldsymbol{R}_{q p} \boldsymbol{\beta}_{i}\right) \\
\boldsymbol{\Gamma}_{\alpha_{i} \beta_{i} \alpha_{i} \beta_{i}} & =\frac{1}{N K^{2}} \sum_{p=1}^{K} \sum_{q=1}^{K}\left(\boldsymbol{\alpha}_{i}^{H} \boldsymbol{R}_{p q} \boldsymbol{\beta}_{i}\right)\left(\boldsymbol{\alpha}_{i}^{H} \boldsymbol{R}_{q p} \boldsymbol{\beta}\right) \\
\boldsymbol{R}_{p q} & =\boldsymbol{R}_{q p}^{H}=E\left[\boldsymbol{h}_{p}(n) \boldsymbol{h}_{q}^{H}(n)\right] .
\end{aligned}
$$

and

Here, $N$ denotes the number of snapshots and $h_{p}$ denotes the data vector at the output of $p$ th subarray.

\section{A. Weighted Smoothing}

In weighted smoothing, the smoothed covariance matrix is obtained by the weighted sum of all the subarray covariance matrices $\boldsymbol{R}=\Sigma_{l=1}^{K} u_{l} \boldsymbol{R}^{l}$, where $u_{l}, l=1,2, \cdots, K$, are the real and positive weights such that $\Sigma_{l=1}^{K} u_{l}=C$, a constant which is positive. By setting the constant to unity one ensures that the source powers which appear as diagonal terms in $S$ are unaltered [5], while off-diagonal terms which represent correlations between the signals are affected. The reason for keeping the weights real and positive is to guarantee the weighted sum of all positive definite matrices to be a positive definite matrix.

The expression in [4] was developed for the uniform weighting case. Recasting this for the general weighting case, we have

$$
\overline{\left|\Delta \theta_{i}\right|^{2}}{ }_{W S}=\left(\frac{\lambda}{2 \pi d \cos \theta_{i}}\right)^{2} \frac{\left[\boldsymbol{\Gamma}_{\alpha_{i} \alpha_{i} \beta_{i} \beta_{i}}^{\prime}+\operatorname{Re} \boldsymbol{\Gamma}_{\alpha_{i} \beta_{i} \alpha_{i} \beta_{i}}^{\prime}\right]}{2\left(\boldsymbol{v}_{1}^{H}\left(\omega_{i}\right) \boldsymbol{P}_{N} \boldsymbol{v}_{1}\left(\omega_{i}\right)\right)^{2}}
$$

where

$$
\boldsymbol{\Gamma}_{\alpha_{i} \alpha_{i} \beta_{i} \beta_{i}}^{\prime}=\frac{1}{N} \sum_{p=1}^{K} \sum_{q=1}^{K} u_{p} u_{q}\left(\alpha_{i}^{H} \boldsymbol{R}_{p q} \boldsymbol{\alpha}_{i}\right)\left(\boldsymbol{\beta}_{i}^{\prime H} \boldsymbol{R}_{q p} \boldsymbol{\beta}_{i}^{\prime}\right)
$$

and

$$
\begin{aligned}
\boldsymbol{\Gamma}_{\alpha_{i} \beta_{i} \alpha_{i} \beta_{i}}^{\prime} & =\frac{1}{N} \sum_{p=1}^{K} \sum_{q=1}^{K} u_{p} u_{q}\left(\boldsymbol{\alpha}_{i}^{H} \boldsymbol{R}_{p q} \boldsymbol{\beta}_{i}^{\prime}\right)\left(\boldsymbol{\alpha}_{i}^{H} \boldsymbol{R}_{q p} \boldsymbol{\beta}_{i}^{\prime}\right) \\
\boldsymbol{\beta}_{i}^{\prime} & =\left(\sum_{l=1}^{K} u_{l} \boldsymbol{A} \Phi^{(l-1)} \boldsymbol{S} \Phi^{-(l-1)} \boldsymbol{A}^{H}\right)^{\#} \boldsymbol{v}\left(\omega_{i}\right)
\end{aligned}
$$

where $(\cdot)^{\#}$ denotes pseudoinverse operation. The weights are chosen so as to minimize the MSE function while constraining them to be real and positive with their sum equal to unity.

\section{B. Optimum Weights}

We now wish to find the optimum weights $u_{l}, l=1,2, \cdots$, $K$, which yield minimum MSE. In general, the problem can be stated as

$$
\begin{gathered}
\min _{\boldsymbol{u}} \boldsymbol{u}^{T} \boldsymbol{Q u} \\
\text { subject to } \quad \boldsymbol{u}^{T} \boldsymbol{e}=1, \quad \boldsymbol{u} \text { is real and positive }
\end{gathered}
$$

where $e=[1,1, \cdots, 1]^{T}$. The $p q$ th element of the matrix $Q$ is given by

$$
[\boldsymbol{Q}]_{p q}=\operatorname{Re}\left\{\left(\boldsymbol{\alpha}_{i}^{H} \boldsymbol{R}_{p q} \boldsymbol{\alpha}_{i}\right)\left(\boldsymbol{\beta}_{i}^{\prime H} \boldsymbol{R}_{q p} \boldsymbol{\beta}_{i}^{\prime}\right)+\left(\boldsymbol{\alpha}_{i}^{H} \boldsymbol{R}_{p q} \boldsymbol{\beta}_{i}^{\prime}\right)\left(\boldsymbol{\alpha}_{i}^{H} \boldsymbol{R}_{q p} \boldsymbol{\beta}_{i}^{\prime}\right)\right\}
$$

A constant factor,

$$
\left(\frac{\lambda}{2 \pi d \cos \theta_{i} \boldsymbol{v}_{1}^{H}\left(\omega_{i}\right) \boldsymbol{P}_{N} \boldsymbol{v}_{1}\left(\omega_{i}\right)}\right)^{2} \frac{1}{2 N}
$$

is not shown in (12) since it will not affect the minimization.
The constrained problem can be recast into an unconstrained problem with the variable substitution

$$
u_{i}=y_{i} y_{i}^{*} /\left(\sum_{l=1}^{K} y_{l} y_{l}^{*}\right), \quad 1 \leq i \leq K
$$

The unconstrained minimization problem is now given by

$$
\min _{y}\left[y_{1} y_{1}^{*}, \cdots, y_{K} y_{K}^{*}\right] \boldsymbol{Q}^{\prime}\left[y_{1} y_{1}^{*}, \cdots, y_{K} y_{K}^{*}\right]^{T}
$$

where $(\cdot)^{*}$ and $(\cdot)^{T}$ denote conjugate and transpose operations, respectively, and the $p q$ th element of $Q^{\prime}$ is given by

$\left[Q^{\prime}\right]_{p q}=\operatorname{Re}\left\{\left(\boldsymbol{\alpha}_{i}^{H} \boldsymbol{R}_{p q} \boldsymbol{\alpha}_{i}\right)\left(\gamma_{i}^{H} \boldsymbol{R}_{q p} \gamma_{i}\right)+\left(\boldsymbol{\alpha}_{i}^{H} \boldsymbol{R}_{p q} \gamma_{i}\right)\left(\boldsymbol{\alpha}_{i}^{H} \boldsymbol{R}_{q p} \gamma_{i}\right)\right\}$

with $\gamma_{i}$ as

$$
\gamma_{i}=\left(\sum_{l=1}^{K} y_{l} y_{l}^{*} \boldsymbol{A} \boldsymbol{\Phi}^{(l-1)} \boldsymbol{S} \boldsymbol{\Phi}^{-(I-1)} \boldsymbol{A}^{H}\right)^{\#} \boldsymbol{v}\left(\omega_{i}\right)
$$

Iterative methods, like the steepest descent method, NewtonRaphson method, and conjugate gradient method, can be used to solve such problems. We have used the steepest descent method to find the optimum weights. The update equation at $n$th iteration is given by

$$
\boldsymbol{y}(n)=\boldsymbol{y}(n-1)-\left.\mu \boldsymbol{g}\right|_{\boldsymbol{y}=\boldsymbol{y}(n-1)}
$$

where $g$ is the gradient vector (of the objective function) with $t$ th element as

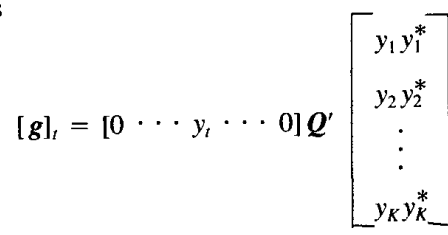$$
+\left[y_{1} y_{1}^{*} \cdots y_{K} y_{K}^{*}\right] \frac{\partial Q^{\prime}}{\partial y_{t}^{*}}\left[\begin{array}{c}
y_{1} y_{1}^{*} \\
y_{2} y_{2}^{*} \\
\vdots \\
y_{K} y_{K}^{*}
\end{array}\right]
$$$$
+\left[y_{1} y_{1}^{*} \cdots y_{K} y_{K}^{*}\right] \boldsymbol{Q}^{\prime}\left[\begin{array}{c}
0 \\
\vdots \\
y_{t} \\
\vdots \\
0
\end{array}\right]
$$

and $\mu$ is a constant representing the step size. The $p q$ th element of $\left(\partial Q^{\prime} / \partial y_{t}^{*}\right)$ is given by

$$
\begin{aligned}
\frac{\partial\left[\boldsymbol{Q}^{\prime}\right]_{p q}}{\partial y_{t}^{*}}= & \left(\boldsymbol{\alpha}_{i}^{H} \boldsymbol{R}_{p q} \boldsymbol{\alpha}_{i}\right)\left(\frac{\partial \gamma_{i}^{H}}{\partial y_{t}^{*}} \boldsymbol{R}_{q p} \gamma_{i}+\gamma_{i}^{H} \boldsymbol{R}_{q p} \frac{\partial \gamma_{i}}{\partial y_{i}^{*}}\right) \\
& +(1 / 2)\left(\boldsymbol{\alpha}_{i}^{H} \boldsymbol{R}_{p q} \frac{\partial \gamma_{i}}{\partial y_{i}^{*}}\right)\left(\boldsymbol{\alpha}_{i}^{H} \boldsymbol{R}_{q p} \gamma_{i}\right) \\
& +(1 / 2)\left(\boldsymbol{\alpha}_{i}^{H} \boldsymbol{R}_{p q} \gamma_{i}\right)\left(\boldsymbol{\alpha}_{i}^{H} \boldsymbol{R}_{q p} \frac{\partial \gamma_{i}}{\partial y_{t}^{*}}\right) \\
& +(1 / 2)\left(\frac{\partial \gamma_{i}^{H}}{\partial y_{t}^{*}} \boldsymbol{R}_{p q} \boldsymbol{\alpha}_{i}\right)\left(\gamma_{i}^{H} \boldsymbol{R}_{q p} \boldsymbol{\alpha}_{i}\right) \\
& +(1 / 2)\left(\gamma_{i}^{H} \boldsymbol{R}_{p q} \boldsymbol{\alpha}_{i}\right)\left(\frac{\partial \gamma_{i}^{H}}{\partial y_{t}^{*}} \boldsymbol{R}_{q p} \boldsymbol{\alpha}_{i}\right)
\end{aligned}
$$




$$
\begin{aligned}
& \text { with } \\
& \begin{aligned}
\frac{\partial \gamma_{i}^{H}}{\partial y_{t}^{*}}= & \left(-\boldsymbol{A}^{H}\right)^{\#}\left(\sum_{l=1}^{K} y_{l} y_{l}^{*} \boldsymbol{\Phi}^{(l-l)} \boldsymbol{S} \boldsymbol{\Phi}^{-(l-1)}\right)^{\#} \\
& \cdot\left(y_{t} \boldsymbol{\Phi}^{(t-1)} \boldsymbol{S} \boldsymbol{\Phi}^{-(t-1)}\right)\left(\sum_{l=1}^{K} y_{l} y_{l}^{*} \boldsymbol{\Phi}^{(l-1)} \boldsymbol{S} \boldsymbol{\Phi}^{-(l-1)}\right)^{\#} \boldsymbol{A}^{\#} \boldsymbol{v}\left(\omega_{i}\right) .
\end{aligned}
\end{aligned}
$$

Once we have the optimum value of $y$, the corresponding optimum value of $\boldsymbol{u}$ can be obtained from (13).

\section{Numerical and Simulation Results}

In this section, we first give some examples to show that the optimum weights yield significantly lower MSE compared to spatial smoothing and then study the tradeoff between the number of subarrays and the subarray size.

In the simulations, 200 independent trials were used to calculate the MSE. The DOA's of the sources were measured with respect to normal to the array, noise power was fixed at $0 \mathrm{~dB}$, and the number of snapshots were fixed at 100 . Throughout this section, we used $-10 \log$ (MSE) as the MSE measure, and therefore, the higher the value of $-10 \log$ (MSE), the lower is the MSE.

\section{A. Optimum Weighting}

We considered a 3-source scenario with DOA's $0^{\circ}, 10^{\circ}$, and $20^{\circ}$, and source powers of $20 \mathrm{~dB}$ each. We studied three different cases with correlation (assuming same value between any two sources) $0.95,0.5$, and 0 . We assumed 7 subarrays with subarray size as 5 . Note that for this subarray size, the DOA separation corresponds to approximately one third of the array beamwidth. The optimum weights were obtained by minimizing the MSE function (formed with true powers, DOA's, and correlations) using the steepest descent method with uniform weights as the initial guess, and the theoretical minimum value of the MSE was determined by evaluating the MSE expression (given in (6)) with the optimum $\boldsymbol{u}$. We may point out here that (6) represents the MSE function for a particular DOA, and hence, the minimization leads to a set of weights that minimize the MSE for the corresponding DOA. However, it has been observed through extensive numerical results that the optimum weights are nearly independent of the choice of DOA.

For the scenarios described above, MSE expression for $10^{\circ}$ was used for determining the optimum weights. Tabel I gives these weights and the values of MSE evaluated from the theoretical expression and the simulations for the root-MUSIC. In the simulations, we used the weights given in the table. The results show that the simulation results are close to those of theoretical, and this validates the use of the theoretical MSE expression. The optimum weighting yields lower MSE compared to the uniform weighting in all the cases, i.e., with $0.95,0.5$, and 0 correlation.

\section{B. Tradeoff Between the Subarray Size and the Number of Subarrays}

In [4], based on the numerical results, it was concluded that the root-MUSIC performs best with full aperture, implying that smoothing at the expense of reduced aperture is not beneficial when the sources are not fully correlated. This, however, is not true as shown by the following example. We considered a 3-source case with DOA's and powers as in the above example and with correlation set to 0.95 . The MSE for different subarray sizes and number of subarrays, keeping the total number of sensors constant at 24 , is calculated for both the optimum and the uniform weighting and plotted the results in Fig. 1. In the figure, we have also included the Cramér-Rao bound (CRB) [6], computed for a 24-element uniform linear array. We note the following from the figure:

1) The MSE without smoothing (which corresponds to $K=1$ ) is about $11 \mathrm{~dB}$ higher than the minimum value that we can obtain with smoothing (uniform and optimum). This establishes the fact that smoothing may be required to improve the performance of the root-MUSIC.

2) The MSE with optimum weighting is close to the CRB for a wider range of number of subarrays compared to the uniform weighting. This shows that we have a wider choice of subarray combinations with optimum weighting.

3) The improvement in the MSE with optimum weighting is larger when the tradeoff is in favor of a greater number of subarrays, as long as the subarray aperture is not too small. In a practical scenario, the number of correlated sources is not known $a$ priori, and therefore, the tradeoff in favor of a greater number of subarrays may be desirable.

In the example considered, the CRB is close to the MSE obtained with spatial smoothing when the number of subarrays is in the range 8 to 14 , and hence, the improvement obtained with the optimum weighting is marginal in this range. However, in the scenarios where the MSE with spatial smoothing is farther away from the CRB, the improvement will be substantial with the optimum weighting.

\section{Methods to Estimate Optimum Weights}

In this section, we show how optimum weights can be estimated when exact knowledge of DOA's, correlations, and powers of sources is not available. This is motivated from the observation that the MSE function is not sensitive to the weights around the optimum point.

We suggest two methods to determine the near-optimum weights. In the first method, the estimates of scenario parameters are obtained from the finite data which are then used in the numerical minimization. In the second method, the optimum weights are approximated with suitable Taylor weights.

\section{A. Method I}

1) Obtain the estimates of DOA's, $\hat{\theta}_{i}, i=1, \cdots, D$, using uniform weighting.

2) Compute the estimates of noise power and signal covariance matrix from $\hat{\sigma}_{\eta}^{2}=$ average of (M-D) smallest eigenvalues of the covariance matrix $\hat{\boldsymbol{R}}$, formed from the total array of $(K+M-1)$ elements, and

$$
\hat{\boldsymbol{S}}=\left(\hat{\boldsymbol{A}}^{H} \hat{\boldsymbol{A}}\right)^{-1} \hat{\boldsymbol{A}}^{H}\left(\hat{\boldsymbol{R}}-\hat{\boldsymbol{\sigma}}_{\eta}^{2} \boldsymbol{I}\right) \hat{\boldsymbol{A}}\left(\hat{\boldsymbol{A}}^{H} \hat{\boldsymbol{A}}\right)^{-1},
$$

where $\hat{\boldsymbol{A}}_{(K+M-1) \times D}$ is the direction matrix formed from the estimated DOA's.

3) Replace the true values with the estimated values and obtain weights by minimizing the MSE function numerically.

For the scenario considered earlier with 0.5 correlation, the estimates of the optimum weights (computed as above) for 5, 7 and 9 subarrays, with subarray size fixed at 5, are shown in Table II. The table gives true optimum weights and its estimates, and theoretical MSE's for these two sets of weights. We should point out here that the estimates of DOA's, noise powers, and signal covariance matrix (steps 1 and 2 ) were obtained from one data realization. Note the closeness between the estimated and true optimum weights and the corresponding MSE's. 
TABLE I

MSE WITH OPTIMUM AND UNIForm WeIghting FOR ROOT-MUSIC (SOURCE DOA's $=0^{\circ}, 10^{\circ}$, AND $20^{\circ}$, SOURCE POWERS $=20$ $\mathrm{dB}$, Noise Power $=0 \mathrm{~dB}$, Subarrays $=7$, Subarray Size $=5$, Snapshots $=100$, Monte Carlo Runs $=200$ )

\begin{tabular}{|c|c|c|c|c|c|}
\hline \multirow{2}{*}{$\begin{array}{l}\text { Correlation } \\
\text { Between Any } \\
\text { Two Sources }\end{array}$} & \multicolumn{2}{|c|}{ Uniform Smoothing } & \multicolumn{3}{|c|}{ Optimum Smoothing } \\
\hline & $\begin{array}{c}\text { Theoretical } \\
-10 \log (\mathrm{MSE})\end{array}$ & $\begin{array}{c}\text { Simulated } \\
-10 \log (\mathrm{MSE})\end{array}$ & $\begin{array}{c}\text { Optimum } \\
\text { Weights }\end{array}$ & $\begin{array}{c}\text { Theoretical } \\
-10 \log \text { (MSE) }\end{array}$ & $\begin{array}{c}\text { Simulated } \\
-10 \log (\text { MSE) }\end{array}$ \\
\hline 0.95 & $11.4156 \mathrm{~dB}$ & $11.5433 \mathrm{~dB}$ & $\begin{array}{l}0.0649 \\
0.0965 \\
0.2157 \\
0.2258 \\
0.2220 \\
0.1051 \\
0.0700\end{array}$ & $\begin{array}{l}13.0566 \mathrm{~dB} \\
\quad(\mathrm{CRB}=22.9730 \mathrm{~dB})\end{array}$ & $13.3761 \mathrm{~dB}$ \\
\hline 0.5 & $18.5328 \mathrm{~dB}$ & $17.4883 \mathrm{~dB}$ & $\begin{array}{l}0.0600 \\
0.1173 \\
0.1991 \\
0.2161 \\
0.2094 \\
0.1295 \\
0.0686\end{array}$ & $\begin{array}{l}23.1846 \mathrm{~dB} \\
\quad(\mathrm{CRB}=30.9540 \mathrm{~dB})\end{array}$ & $22.4108 \mathrm{~dB}$ \\
\hline 0 & $20.0867 \mathrm{~dB}$ & $20.4337 \mathrm{~dB}$ & $\begin{array}{l}0.0708 \\
0.1264 \\
0.1998 \\
0.2060 \\
0.1948 \\
0.1264 \\
0.0708\end{array}$ & $\begin{array}{l}23.7179 \mathrm{~dB} \\
\quad(\mathrm{CRB}=32.4347 \mathrm{~dB})\end{array}$ & $24.2277 \mathrm{~dB}$ \\
\hline
\end{tabular}

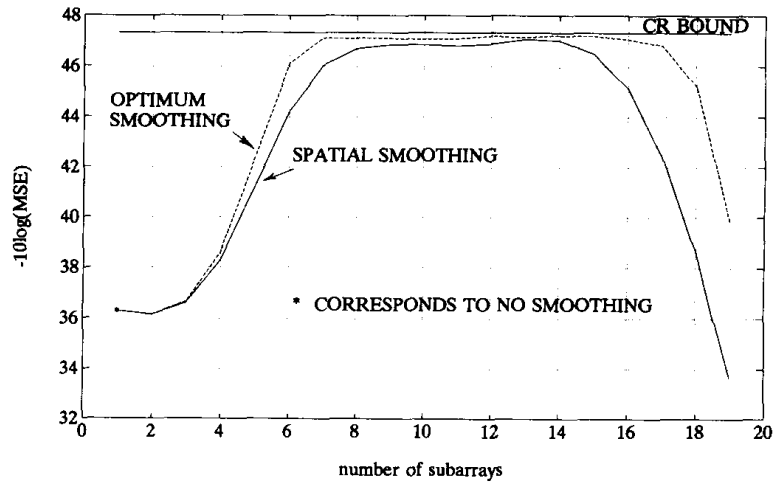

Fig. 1. Optimum tradeoff between subarray size and number of subarrays for root-MUSIC (source DOA's: $0^{\circ}, 10^{\circ}$, and $20^{\circ}$, source powers $=20$ $\mathrm{dB}$, noise power $=0 \mathrm{~dB}$, correlation between any two sources $=0.95$ total array size $=24$, number of snapshots $=100$ ).

\section{B. Method II}

In this section we show how Taylor weights can be used as nearoptimum weights. The computation of Taylor weights [7] requires three parameters: number of weights, number of sidelobes having equal amplitude, and the desired sidelobe level. We have chosen the number of weights as the number of subarrays and the number of sidelobes having equal amplitude as $2,{ }^{\prime}$ and determined an appropriate sidelobe level for which the Taylor weights approximate the optimum weights in the mean-square sense.

'This number was arrived at through inspection and was found to give better results than the others.
For the 3-source uncorrelated scenario used in Section III-A, we computed the optimum weights and determined the sidelobe level such that the corresponding Taylor weights were closest to the optimum weights in the mean-square sense. This sidelobe level was found to be $-38 \mathrm{~dB}$. Table III gives the theoretical values of MSE with uniform weights, optimum weights, and $-38 \mathrm{~dB}$ Taylor weights for various combinations of DOA's, source powers (assumed to be equal), and correlations. Note that the Taylor weights were fixed in all the cases while the optimum weights were determined for each scenario.

The results show that the MSE performance with Taylor weights is very close to that with optimum weights in all the scenarios, implying that the Taylor weights determined from the approximate knowledge of the scenario serve as near-optimum weights for a range of scenarios that are moderately different from the approximate.

\section{Conclusions}

We have considered a generalized weighting in the subarray covariance averaging problem, and presented a method to obtain optimum weights which give minimum MSE in the DOA estimates. Since the computation of optimum weights requires a priori knowledge of scenario parameters, we suggested methods to determine near-optimum weights. Using numerical results we have shown that the near-optimum weights perform as good as the optimum weights, and they can be determined from the approximate knowledge of the scenario.

\section{ACKNOWLEDGMENT}

The authors are grateful to Dr. K. V. S. Hari for his useful comments during the preparation of the manuscript. 
TABLE II

MSE Values with the True Optimum Weights and Their Estimates for the Case of Root-MUSiC (Source DOA's: $0^{\circ}$, $10^{\circ}$, and $20^{\circ}$, Source Powers $=20 \mathrm{~dB}$, Noise power $=0 \mathrm{~dB}$, Correlation Between any Two Sources $=0.5$, Subarray $10^{\circ}$, AND $20^{\circ}$, SOURCE POWERS $=20 \mathrm{~dB}$, NOISE POWER $=0 \mathrm{~dB}$, CORRELATION
SIZE $=5$, SNAPSHOTS $=100$ )

\begin{tabular}{clcr}
$\begin{array}{l}\text { Number of } \\
\text { Subarrays }\end{array}$ & & Weights & $\begin{array}{c}\text { Theoretical } \\
10 \text { log (MSE) }\end{array}$ \\
\hline \multirow{2}{*}{5} & True & $0.1221,0.2138,0.2994,0.2267,0.1300$ & $18.7981 \mathrm{~dB}$ \\
& Estimates & $0.1224,0.2356,0.3262,0.2054,0.1103$ & $18.3815 \mathrm{~dB}$ \\
7 & True & $0.0600,0.1173,0.1991,0.2161,0.2094,0.1295,0.0686$ & $23.1846 \mathrm{~dB}$ \\
& Estimates & $0.0640,0.1206,0.2145,0.2196,0.2147,0.1118,0.0549$ & $22.9569 \mathrm{~dB}$ \\
9 & True & $0.0391,0.0776,0.1350,0.1632,0.1842,0.1599,0.1300$, & $25.8056 \mathrm{~dB}$ \\
& Estimates & $0.0739,0.0371$ & $0.0414,0.0800,0.1366,0.1622,0.1805,0.1489,0.1333$, \\
& & $0.0756,0.0413$ & $25.7258 \mathrm{~dB}$ \\
\hline
\end{tabular}

TABLE III

Theoretical MSE with Uniform, Taylor $(-38 \mathrm{~dB}$ ), and Optimum Weights for Root-MUSIC (Noise POWeR $=0 \mathrm{~dB}$ SUbarrays $=7$, Subarray Size $=5$, SNAPShots $=100$ )

\begin{tabular}{|c|c|c|c|c|c|}
\hline DOA's & $\begin{array}{l}\text { Correlation } \\
\text { Between Any } \\
\text { Two Sources }\end{array}$ & $\begin{array}{l}\text { Source } \\
\text { Powers }\end{array}$ & $\begin{array}{c}\text { Uniform } \\
\text { Weighting } \\
-10 \log (\mathrm{MSE})\end{array}$ & $\begin{array}{c}\text { Taylor } \\
\text { Weighting } \\
-10 \log (\text { MSE) }\end{array}$ & $\begin{array}{c}\text { Optimum } \\
\text { Weighting } \\
-10 \log \text { (MSE) }\end{array}$ \\
\hline \multirow[t]{2}{*}{$\begin{array}{l}0^{\circ}, 10^{\circ} \text {, } \\
\quad \text { and } 20^{\circ}\end{array}$} & 0.5 & $\begin{array}{l}30 \mathrm{~dB} \\
20 \mathrm{~dB} \\
10 \mathrm{~dB}\end{array}$ & $\begin{array}{l}29.1333 \mathrm{~dB} \\
18.5328 \mathrm{~dB} \\
04.9319 \mathrm{~dB}\end{array}$ & $\begin{array}{l}35.5223 \mathrm{~dB} \\
22.9606 \mathrm{~dB} \\
05.5921 \mathrm{~dB}\end{array}$ & $\begin{array}{l}35.9374 \mathrm{~dB} \\
23.1846 \mathrm{~dB} \\
05.9399 \mathrm{~dB}\end{array}$ \\
\hline & 0 & $\begin{array}{l}30 \mathrm{~dB} \\
20 \mathrm{~dB} \\
10 \mathrm{~dB}\end{array}$ & $\begin{array}{l}31.5506 \mathrm{~dB} \\
20.0867 \mathrm{~dB} \\
04.2192 \mathrm{~dB}\end{array}$ & $\begin{array}{l}39.3925 \mathrm{~dB} \\
23.4805 \mathrm{~dB} \\
04.2193 \mathrm{~dB}\end{array}$ & $\begin{array}{l}40.2570 \mathrm{~dB} \\
23.7178 \mathrm{~dB} \\
04.5455 \mathrm{~dB}\end{array}$ \\
\hline \multirow[t]{2}{*}{$\begin{array}{l}0^{\circ}, 15^{\circ}, \\
\text { and } 30^{\circ}\end{array}$} & 0.5 & $\begin{array}{l}30 \mathrm{~dB} \\
20 \mathrm{~dB} \\
10 \mathrm{~dB}\end{array}$ & $\begin{array}{l}38.1907 \mathrm{~dB} \\
27.9937 \mathrm{~dB} \\
16.4000 \mathrm{~dB}\end{array}$ & $\begin{array}{l}43.4277 \mathrm{~dB} \\
32.8244 \mathrm{~dB} \\
19.2125 \mathrm{~dB}\end{array}$ & $\begin{array}{l}43.7572 \mathrm{~dB} \\
33.1157 \mathrm{~dB} \\
19.5458 \mathrm{~dB}\end{array}$ \\
\hline & 0 & $\begin{array}{l}30 \mathrm{~dB} \\
20 \mathrm{~dB} \\
10 \mathrm{~dB}\end{array}$ & $\begin{array}{l}39.0146 \mathrm{~dB} \\
28.7374 \mathrm{~dB} \\
16.6468 \mathrm{~dB}\end{array}$ & $\begin{array}{l}46.5696 \mathrm{~dB} \\
34.9904 \mathrm{~dB} \\
18.9175 \mathrm{~dB}\end{array}$ & $\begin{array}{l}47.5345 \mathrm{~dB} \\
35.6143 \mathrm{~dB} \\
19.0794 \mathrm{~dB}\end{array}$ \\
\hline
\end{tabular}

\section{REFERENCES}

[1] T. J. Shan, M. Wax, and T. Kailath, "On spatial smoothing for direction-of-arrival estimation of coherent signals," IEEE Trans. Acoust., Speech, Signal Processing, vol. 33, no. 4, pp. 806-811, Aug. 1985.

[2] A. Paulraj, V. U. Reddy, T. J. Shan, and T. Kailath, "Performance analysis of the MUSIC algorithm with spatial smoothing in the presence of coherent sources," in Proc. MILCOM, 1986, pp. 41.5.141.5.5.

[3] M. Kaveh and A. J. Barabell, "Statistical performance analysis of the MUSIC and min-norm algorithm in resolving plane waves in noise," IEEE Trans. Acoust., Speech, Signal Processing, vol. 34, pp. 331 $341,1986$.

[4] B. D. Rao, and K. V. S. Hari, "Effect of spatial smoothing on the performance of MUSIC and the Minimum-norm method," Proc. Inst. Elec. Eng., vol. 137, pt. F, no. 6, pp. 449-458, Dec. 1990

[5] A. Paulraj, V. U. Reddy, and T. Kailath, "Analysis of signal cancellation due to multipath in optimum beamformers for moving arrays," IEEE J. Ocean. Eng., vol. 12, pp. 163-172, Jan. 1987.

[6] P. Stoica and A. Nehorai, "MUSIC, maximum likelihood, and Cramér-Rao bound," IEEE Trans. Acoust., Speech, Signal Processing, vol. 37, pp. 720-741, May 1989.

[7] A. T. Villeneuve, "Taylor pattern for discrete arrays," IEEE Trans. Antennas Propagat., pp. 1089-1093, Oct. 1984

[8] S. U. Pillai and B. H. Kwon, "Performance analysis of MUSIC-type high resolution estimators for direction finding in correlated and coherent scenes," IEEE Trans. Acoust., Speech, Signal Processing, pp. 1176-1189, Aug. 1989

[9] A. J. Barabell, "Improving the resolution performance of eigenstruc- ture-based direction-finding algorithms,"' in Proc. ICASSP 83, 1983, pp. 336-339.

\section{Observation Noise and Zero Loci of a Time Series Model}

Kuniharu Kishida, Sumasu Yamada, and Nobuo Sugibayashi

Abstract-The properties of zeros of time series models are examined in a linear stochastic system with white Gaussian observation noise. Each zero has a locus in the complex plane as the variance of obser-

Manuscript received May 20, 1991; revised August 21, 1992. The associate editor coordinating the review of this correspondence and approving it for publication was Dr. David Rossi. This work was supported in part by a Grant-in-Aid for Scientific Research 03808026 from the Ministry of Education, Science, and Culture of Japan.

K. Kishida is with the Department of Applied Mathematics, Faculty of Engineering, Gifu University, Yanagido, Gifu 501-11, Japan.

S. Yamada is with the Department of Electrical Engineering. Faculty of Engineering, Setsunan University, Neyagawa 572 , Japan.

N. Sugibayashi is with the Mitsubishi Electric Corporation, Power and Industrial System Center, Kobe, Hyogoken 652, Japan.

IEEE Log Number 9208206. 